\title{
Hypothermia and erythropoietin for neuroprotection after neonatal brain damage
}

\author{
Xiyong Fan',2, Frank van Bel' ${ }^{1}$, Michael A. van der Kooij ${ }^{1,2}$, Cobi J. Heijnen ${ }^{2}$ and Floris Groenendaal'
}

BACKGROUND: Both hypothermia and erythropoietin (EPO) are reported to have neuroprotective effects after perinatal hypoxia-ischemia (HI). We investigated a possible additive effect of the use of a combination of hypothermia-EPO in a rat model of neonatal $\mathrm{HI}$.

METHODS: At postnatal day 7, rats were subjected to HI and then randomized to $3 \mathrm{~h}$ of hypothermia, EPO, or both. Sensorimotor function was assessed by the cylinder-rearing test (CRT) at 2 and $5 \mathrm{wk}$ after $\mathrm{HI}$. Brain lesion volume and white matter loss were determined by hematoxylin-eosin and luxol fast blue staining, respectively.

RESULTS: Multivariable analysis using general linear modeling showed that hypothermia, EPO, and the interaction hypothermia $\times$ gender were determinants of sensorimotor function, both at 2 and 5 wk after $\mathrm{HI}$. Neuroprotective effects of hypothermia at 5 wk were more pronounced in females, showing 52\% improvement in the CRT. Maximal improvement in males was $26 \%$ after combined treatment with hypothermia and EPO. Histological outcome was improved by hypothermia only with no additional effect of EPO or gender.

CONCLUSION: Hypothermia after HI improved sensorimotor function in females more than in males. There was a borderline additive effect of EPO when combined with hypothermia. Histology of brain lesion volume and white matter damage was improved only by hypothermia.

$\mathbf{P}$ erinatal hypoxia-ischemia (HI) is an important cause of neonatal brain injury and is associated with long-term neurological sequelae such as cognitive dysfunction, developmental delay, seizures, and sensory and/or motor impairment $(1,2)$. Several studies in newborn rodents indicate that reduction of brain temperature by $2-5^{\circ} \mathrm{C}$ even for $3 \mathrm{~h}$, when started within $6 \mathrm{~h}$ after $\mathrm{HI}$, provides neuroprotection and improved behavioral outcome (3-6).

The neuroprotective effects of hypothermia of mildly asphyxiated newborns have been demonstrated, but in clinical practice, morbidity and mortality remain considerable (30-45\%) $(7,8)$. The combination of hypothermia and other pharmacologic strategies after birth asphyxia may improve long-term neurodevelopment (9).
Erythropoietin (EPO) has been shown to reduce brain lesion volume in an experimental setting of neonatal HI (10-13). In addition, EPO treatment of perinatally asphyxiated human term neonates has also proven to be beneficial (14). In recent studies with a rodent model, females appear to benefit more from neuroprotective interventions after $\mathrm{HI}$, such as hypothermia, EPO, and 2-iminobiotin (15-17).

The aim of the present study was to test whether the addition of EPO to hypothermia has additive neuroprotective effects as compared with a single treatment and to examine possible gender effects.

\section{RESULTS}

Hypothermia

Sham-treated rats did not show a paw preference in the cylinder-rearing test (CRT) (paw preference $< \pm 5 \%$, data not shown). A significant paw preference of the unimpaired forepaw in the normothermia groups was observed at both 2 and $5 \mathrm{wk}$ after HI (Figure 1). Differences between males and females in the normothermia group were not statistically significant. In female rats, hypothermia alone reduced paw preference in the CRT from $69 \pm 13 \%$ to $31 \pm 13 \%$ at $2 \mathrm{wk}$ and from $45 \pm 12 \%$ to $19 \pm 12 \%$ at $5 \mathrm{wk}$, whereas in males, the corresponding values were $65 \pm 13 \%$ to $55 \pm 12 \%$ and $41 \pm 12 \%$ to $34 \pm 12 \%$, respectively.

The effect of hypothermia on lesion volume was determined at $5 \mathrm{wk}$ after HI. The morphological changes in the brain as visualized after hematoxylin-eosin (HE) staining are shown in Figure 2a. There was a $75 \%$ loss of tissue at the ipsilateral hemisphere after HI. The lesion volumes were similar in male and female rats. At the contralateral hemisphere after HI, there were no statistically significant changes in the volume of cortex, hippocampus, and white matter as compared with the sham-treated animals (Figure 2e-g). Hypothermia increased the ipsilateral/contralateral brain volume ratio by $\sim 20 \%$ in females $(P<0.05)$, but not in males (Figure 2c). Similar effects were observed when analyzing white matter damage (luxol fast blue (LFB) staining, $P<0.05$, Figure $2 \mathrm{~d}$ ).

\section{EPO}

At 2 and $5 \mathrm{wk}$ after HI, EPO mildly improved the sensorimotor function as shown by the reduction of paw preference during 

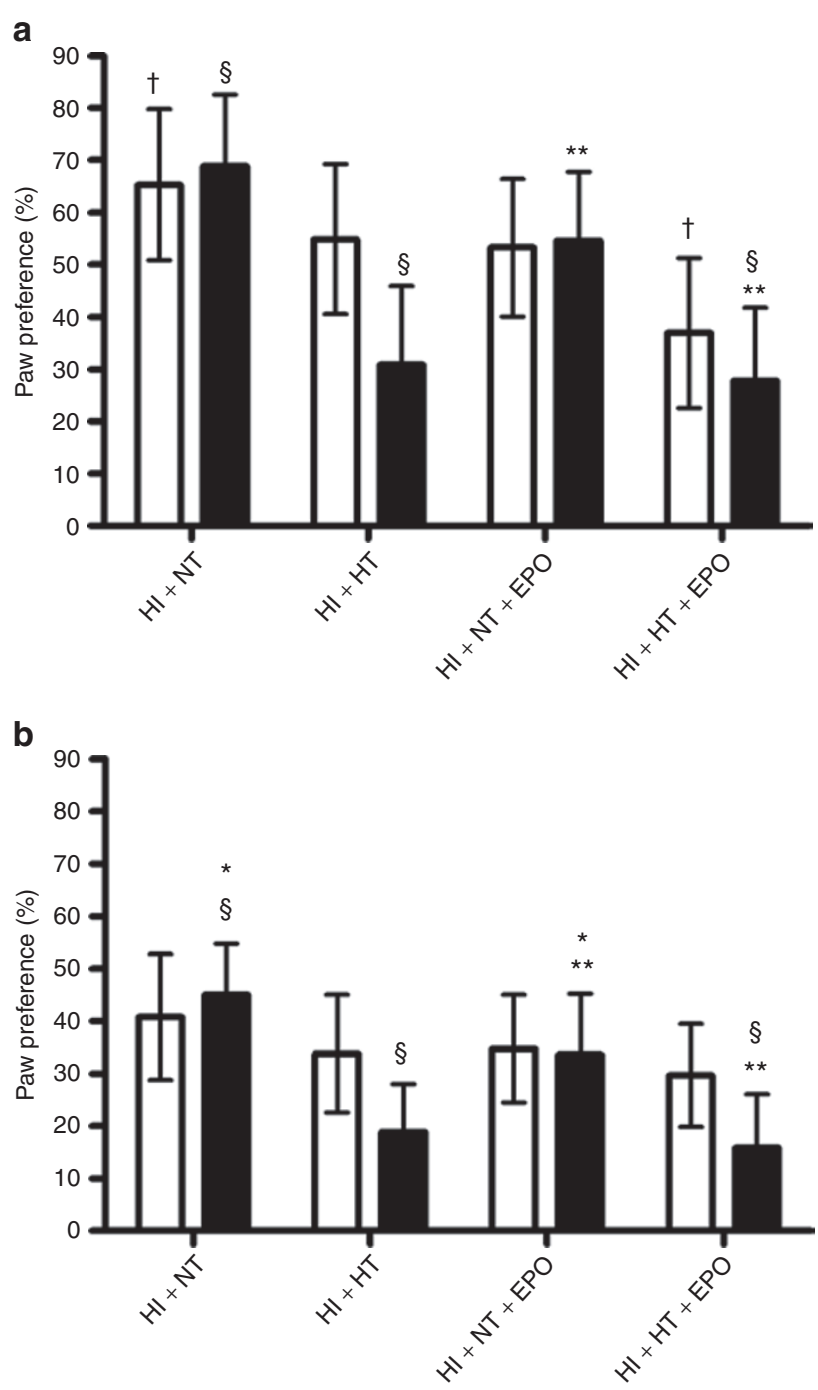

Figure 1. Effect of hypothermia and EPO on preference to use the nonimpaired (ipsilateral) paw in the cylinder-rearing test. Male (open bars) and female (filled bars) P7 rats were exposed to $\mathrm{HI}$ and treated with $3 \mathrm{~h}$ hypothermia or normothermia, combined with EPO $(5 \mathrm{kU} / \mathrm{kg})$ or vehicle. At (a) $2 \mathrm{wk}$ and (b) $5 \mathrm{wk}$ after $\mathrm{HI}$, sensorimotor function was assessed in the cylinder-rearing test. The relative nonimpaired forepaw (right, ipsilateral) preference was calculated as (right - left)/(right + left + both) $\times 100 .{ }^{5} \mathrm{HI}+$ NT vs. $\mathrm{HI}+\mathrm{HT}, \mathrm{HI}+\mathrm{NT}$ vs. $\mathrm{HI}+\mathrm{HT}+\mathrm{EPO}$ in females, $P<0.001{ }^{* *} \mathrm{HI}+\mathrm{NT}+$ $\mathrm{EPO}$ vs. $\mathrm{HI}+\mathrm{HT}+\mathrm{EPO}$ in females, $\mathrm{P}<0.01 .{ }^{+} \mathrm{HI}+\mathrm{NT}$ vs. $\mathrm{HI}+\mathrm{HT}+\mathrm{EPO}$ in males, $P<0.01$. ${ }^{*} \mathrm{HI}+\mathrm{NT}$ vs. $\mathrm{HI}+\mathrm{NT}+$ EPO in females, $P<0.05 ; n=13$ per group. EPO, erythropoietin; $\mathrm{HI}$, hypoxia-ischemia; $\mathrm{HT}$, hypothermia; NT, normothermia; P, postnatal day.

CRT (Figure 1). EPO showed no effect on brain tissue loss and white matter damage in either males or females with respect to HE staining or LFB staining (Figure 2).

\section{Hypothermia-EPO Combination}

Addition of EPO to hypothermia improved sensorimotor function in males at $2 \mathrm{wk}$ after HI from $65 \pm 13 \%$ to 37 $\pm 13 \%$ (Figure 1a), and from $41 \pm 12 \%$ to $30 \pm 12 \%$ at $5 \mathrm{wk}$ (Figure 1b). In females, paw preference was reduced from 69 $\pm 13 \%$ to $28 \pm 12 \%$ at $2 \mathrm{wk}$ and from $45 \pm 12 \%$ to $16 \pm 12 \%$ at 5 wk after HI.
The addition of EPO to hypothermia did not decrease lesion volume as shown by HE staining, nor did it improve white matter lesions.

\section{Multivariable Analysis}

Multivariable analysis using general linear modeling showed that hypothermia $(P=0.0002)$, EPO $(P=0.07)$, and the interaction hypothermia $\times$ gender $(P=0.02)$ were determinants of sensorimotor function, both at $2 \mathrm{wk}$ (data not shown) and $5 \mathrm{wk}$ after HI (Table 1). The model demonstrates that $52 \%$ improvement in the CRT could be achieved at $5 \mathrm{wk}$ in females by hypothermia alone, whereas in males a maximum of $26 \%$ improvement could be obtained by the combination of hypothermia and EPO. Histological outcome was improved only by hypothermia $(P=0.02)$ with no additional effect of EPO or gender.

\section{DISCUSSION}

In this study, we examined the neuroprotective effects of moderate hypothermia with or without EPO after HI in rat pups. Three hours of moderate hypothermia provided extensive long-term neuroprotection in female rats, with very modest effects in males. This duration of hypothermia has previously been shown to be sufficient for neuroprotection, while avoiding the risks of hypoglycemia (18).

Several in vitro and in vivo studies have demonstrated the neuroprotective properties of hypothermia on changes of inflammatory and apoptotic markers in differentiated PC12 cells and in a rat model of neonatal HI $(19,20)$. The improvement of long-term motor behavior after hypothermia was also demonstrated in a rat model of neonatal HI (4). To date, gender effects of hypothermia were reported only by Bona et al. (15), who observed more effective neuroprotection in female rats than in male rats. In the present study, we observed strong neuroprotective effects of hypothermia in female rats but saw weaker effects in male rats.

The mechanisms of action of hypothermia as a therapy for neonatal hypoxic-ischemic brain injury are still under investigation. More and more studies consider the main benefit from hypothermia to be a delay in the expression of brain damage, thereby extending the therapeutic window and allowing additional therapeutic interventions after $\mathrm{HI}(18,21,22)$.

Before investigating the effects of the hypothermia-EPO combination, we tested the effect of EPO on recovery after HI. In the present study, EPO alone provided a mild improvement of sensorimotor function. Effects were too small to demonstrate any gender effect with multiple regression analysis in the present study, although some studies in both animals and humans suggest that the neuroprotective effect of EPO may be gender dependent $(14,17)$. EPO has been reported to have neuroprotective abilities via anti-inflammation, antiapoptosis, and neuroregeneration $(10,11,23)$. Recently, EPO has been used in relatively high dosages $(0.5-3 \mathrm{kU} / \mathrm{kg})$ for at least three times in (preterm) infants, and improvement of long-term outcome was observed (24-26). Using the same animal model, a previous study from our group found no neuroprotective effect of EPO when administered at $72 \mathrm{~h}$ 
a

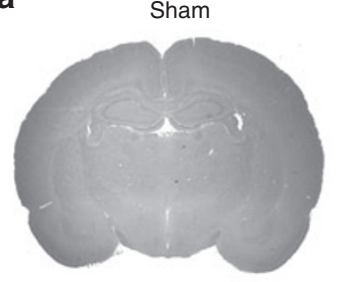

$\mathrm{HI}+\mathrm{NT}$

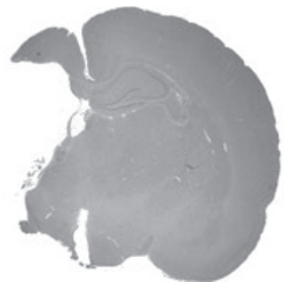

$\mathrm{HI}+\mathrm{NT}$
$\mathrm{HI}+\mathrm{HT}$

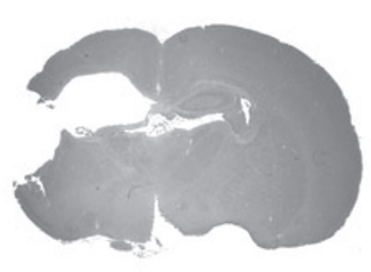

$\mathrm{HI}+\mathrm{HT}$
$\mathrm{HI}+\mathrm{NT}+\mathrm{EPO}$

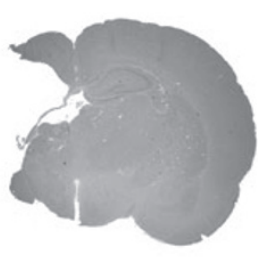

$\mathrm{HI}+\mathrm{NT}+\mathrm{EPO}$

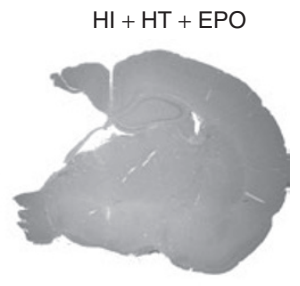

b Sham
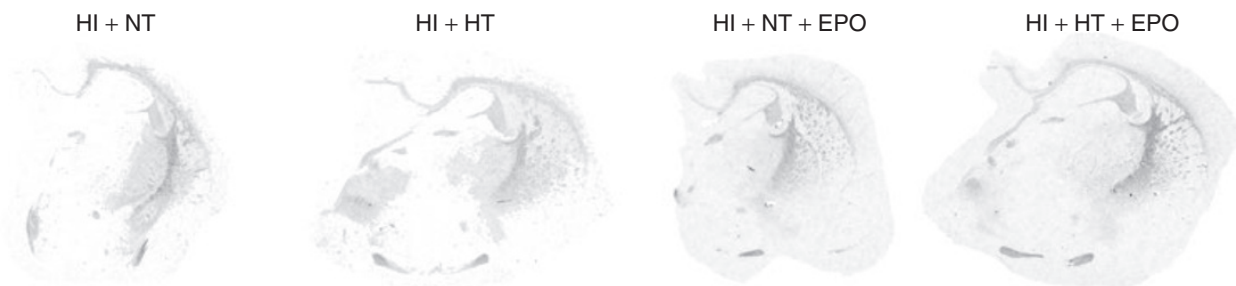

C
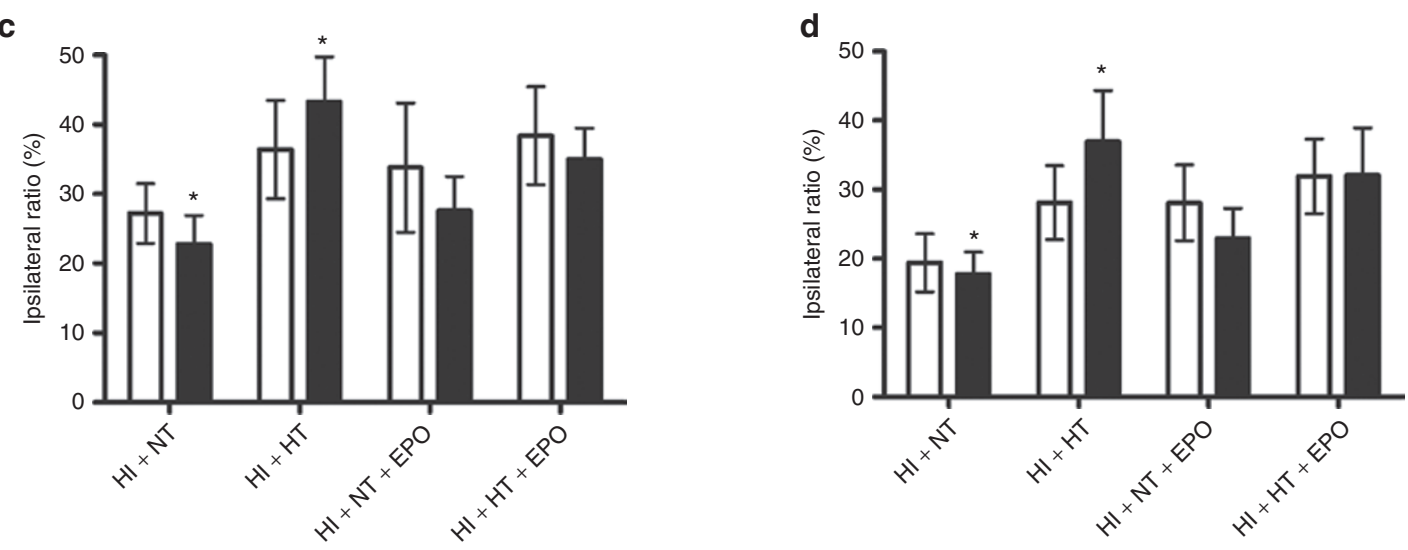

e

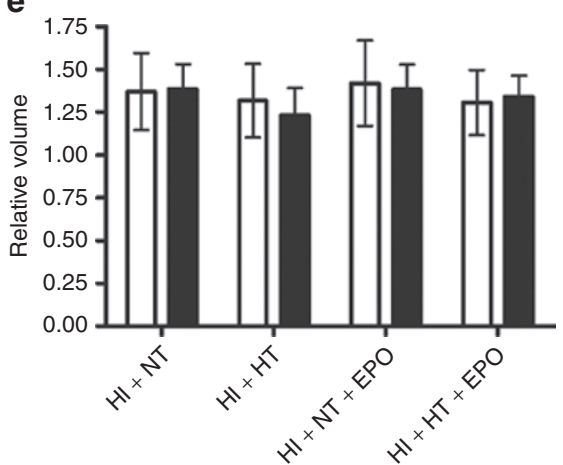

f

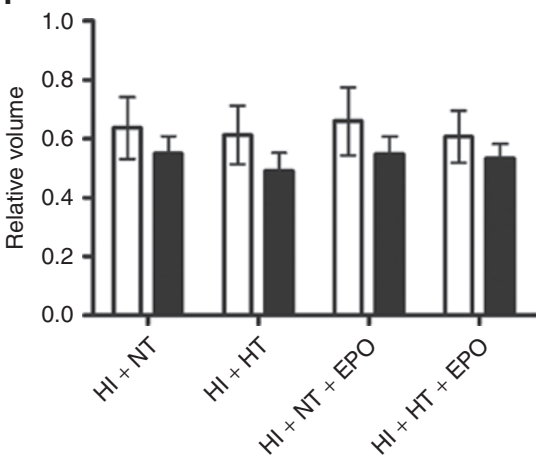

g

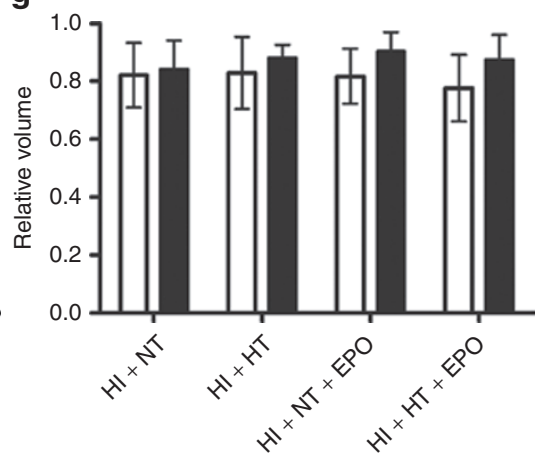

Figure 2. Effect of hypothermia and EPO treatment on brain area loss and white matter loss as determined after hematoxylin and eosin (HE) and luxol fast blue (LFB) staining, respectively, at $5 \mathrm{wk}$ after HI. (a) Representative examples of brain area loss after HE staining (examples of sham + NT + EPO and sham + $\mathrm{HT}+\mathrm{EPO}$ groups are similar to those of the sham group, data not shown here). (b) Representative examples of white matter changes of the brain after LFB staining. Sham + NT + EPO and sham + HT + EPO groups were similar to the sham group (data not shown). (c) The ratio of ipsilateral/contralateral areas in male (open bars) and female (filled bars) rats after HE staining. (d) The white matter ratio of ipsilateral/contralateral areas in male (open bars) and female (filled bars) rats. The ipsilateral/contralateral ratio in $\mathbf{c}$ and $\mathbf{d}$ of the sham group is around $100 \%$ (data not shown). (e) The relative volume (sham $=1$ ) of contralateral cortex in male (open bars) and female (filled bars) rats. (f) The relative volume (sham $=1$ ) of contralateral hippocampus in male (open bars) and female (filled bars) rats. (g) The relative volume of contralateral white matter in male (open bars) and female (filled bars) rats. ${ }^{*} \mathrm{HI}+\mathrm{NT}$ vs. $\mathrm{HI}+\mathrm{HT}$ in females, $P<0.05 ; n=13$ per group (sham group $n=4$ ). EPO, erythropoietin; HI, hypoxia-ischemia; HT, hypothermia; NT, normothermia.

after HI (12). However, Spandou et al. (27) reported that, in a similar rat model using a shorter duration of hypoxia, EPO effectively reduced brain tissue loss. We cannot exclude the possibility that EPO provides neuroprotective effects after milder brain injury.
Our study is the first to examine neuroprotective effects of combined hypothermia-EPO in newborn animals with HI. The combination of EPO and hypothermia has been reported in adult patients after out-of-hospital cardiac arrest (28), and administration of EPO further increased the survival rate of 
Table 1. Multiple regression analysis of paw preference in the cylinder-rearing test at $5 \mathrm{wk}$ after hypoxia-ischemia

\begin{tabular}{lccc}
\hline & Coefficient & SEM & $P$ value \\
\hline Intercept & 42.3 & 3.9 & $<2 \times 10^{-16}$ \\
Gender & -1.5 & 4.8 & 0.76 \\
Hypothermia & -21.8 & 4.9 & 0.0002 \\
EPO & -6.2 & 3.4 & 0.07 \\
Interaction gender $\times$ & 15.9 & 6.8 & 0.02 \\
hypothermia & & &
\end{tabular}

Estimates of paw preference (\%). Gender ( $0=$ female, $1=$ male), hypothermia $(0=$ no, $1=$ yes $)$, EPO (0 = no, 1 =yes $)$.

EPO, erythropoietin.

patients after hypothermia treatment. Adding EPO to hypothermia had only a borderline neuroprotective effect in the present study $(P=0.07)$. In males, the magnitude of the effect of EPO in the CRT was of the same size as the effect of hypothermia. In combination, $26 \%$ improvement was obtained.

To explain this observation, the neuroprotective mechanisms of these two interventions should be considered. Recently, both hypothermia and EPO have been recognized to reduce free radical formation and apoptotic activity and to inhibit inflammatory response $(5,11)$, but the comparison of the effects between hypothermia and EPO has not been reported. In our study, single treatment with hypothermia has been shown to have a neuroprotective effect in females, and is probably powerful enough to block the secondary energy failure, leaving no potential for EPO to be effective during simultaneous administration. This does not exclude the possibility that beneficial effects of delayed administration of EPO might be possible after extending the therapeutic window through hypothermia. Delayed and prolonged administration of EPO has shown beneficial effects on functional recovery $(23,29)$. Of note, no reduction in brain lesion volume after delayed treatment with EPO was found, but improvement in sensorimotor neurological function and white matter regeneration was observed (29). Also in our study, functional recovery was much more pronounced than histological recovery.

In the present study, we focused on the ipsilateral hemisphere. In previous experiments, we have demonstrated the absence of long-term histological changes in the contralateral hemisphere in this animal model (30). This study also indicates that there were no statistical differences between the relative volume of the contralateral cortex, hippocampus, and white matter of the different treatment groups.

This study also revealed gender differences in response to the hypothermia and treatment with EPO. Differences in apoptotic mechanisms may have played a role in the observed gender differences. It has been reported previously that pathways of apoptotic cell death in males differ from the pathways in females. Both in vitro and in vivo studies indicated that females predominantly use the caspase-dependent pathway of apoptosis whereas males merely use the caspase-independent pathway by translocation of apoptosis-inducing factor from mitochondria to the nucleus $(16,31,32)$. In animal models of neonatal $\mathrm{HI}$, reduction in caspase- 3 activity has been shown after either EPO or hypothermia treatment $(5,12)$.

Spontaneous improvement of sensorimotor function was observed in our experiments. This has been described previously and may be considered a result of plasticity of the brain (33). Plasticity is one of the most prominent features of the central nervous system, especially in the developing brain (34), and EPO has been reported to promote brain plasticity in both newborn and adult animal models of brain injury $(29,35)$. In the present study, although the animals after HI can benefit from the plasticity of the central nervous system, further improvement of sensorimotor function can be obtained from the treatment with EPO or the hypothermia-EPO combination.

Finally, we cannot exclude the possibility that the combination of hypothermia-EPO is more effective in milder models of $\mathrm{HI}$. In this study, we have used an animal model of severe neonatal $\mathrm{HI}$ to mimic the clinical aspects of perinatal asphyxia $(7,8)$.

In conclusion, hypothermia after $\mathrm{HI}$ has long-lasting neuroprotective effects, which are much more pronounced in female rats. In male rats, the combination of hypothermia with EPO showed a $26 \%$ improvement of sensorimotor function. The hypothermia-EPO combination did not show improvement of lesion volume or of white matter damage.

\section{METHODS}

\section{Animals}

Experiments were performed in accordance with international guidelines and approved by the Animal Care Committee of the University Medical Center Utrecht. Wistar rats were bred at the animal facility of the University of Utrecht. Experiments were performed at postnatal day 7 (P7). Rats were housed on a normal day-night cycle, weaned at the age of $4 \mathrm{wk}$, and housed singly for the remainder of the experiment. Food and water were available ad libitum. Treatments were evenly distributed over the litters and randomly chosen per animal.

\section{Experimental Model}

The well-known Rice-Vannucci model of perinatal cerebral HI was used in our experiments (36). P7 pups were anesthetized (isoflurane, $5 \%$ induction/1.5\% maintenance in $\mathrm{O}_{2}: \mathrm{N}_{2} \mathrm{O} ; 1: 1$ ) and the right common carotid artery was ligated. After $1.5 \mathrm{~h}$ of recovery, rats were subjected to $8 \% \mathrm{O}_{2}$ in $\mathrm{N}_{2}$ for $90 \mathrm{~min}(n=26)$ at a temperature of $37^{\circ} \mathrm{C}(36)$. Shamtreated controls were anesthesia and skin incision but no artery occlusion or hypoxia (12 males and 12 females).

A total of 110 rat pups (55 males and 55 females) underwent unilateral HI. Six rats (3 males and 3 females) (5.5\%) died during hypoxia before randomization for treatment, but no deaths occurred after HI insult.

\section{Hypothermia}

Hypothermia was started immediately after hypoxia. Animals (including eight sham-treated animals) randomized for treatment were placed into an infant incubator for $3 \mathrm{~h}$ (30 males and 30 females). In the same incubator, additional animals, the so called "sentinel" pups (gender randomized) were also placed. From the latter, temperature was continuously measured using a BIO-BRET- 4 calibrated $\left(<0.1^{\circ} \mathrm{C}\right.$ deviation) temperature probe (Bioseb, Vitrolles, France) (18). These sentinel pups were excluded from further analysis and killed immediately after hypothermia, as the stress of carrying a probe may affect outcome (18). The incubator temperature setting was adjusted to keep the rectal temperature between 32.5 and $33^{\circ} \mathrm{C}$. Rectal temperature was recorded every $5 \mathrm{~min}$. Animals (including eight sham-treated animals) randomized for normothermia treatment were placed in another infant incubator (30 males and 30 females), and the rectal temperature, which was sustained between 36.5 and $37^{\circ} \mathrm{C}$, was measured in the same way (Figure 3). 


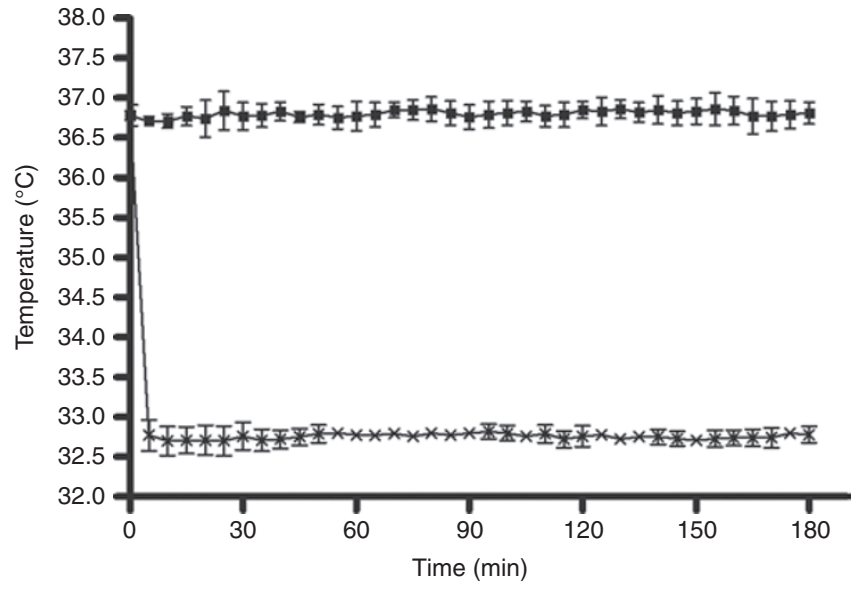

Figure 3. The graph shows rectal temperature (mean $\pm \mathrm{SD}$ ) of the so called "sentinel" rats $(n=10)$ in an infant incubator by $3 \mathrm{~h}$ intervention of normothermia (filled squares, $37^{\circ} \mathrm{C}$ ) or hypothermia (multiplication symbols, $\left.32.5-33^{\circ} \mathrm{C}\right)$.

\section{EPO Administration}

EPREX EPO (Janssen-Cilag B.V., Tilburg, Netherlands) was diluted in $0.9 \% \mathrm{NaCl}$ and injected intraperitoneally (i.p.) at $5 \mathrm{kU} / \mathrm{kg}$ (volume: $10 \mathrm{ml} / \mathrm{kg}$ ) immediately after hypothermia, with two additional doses given at $24 \mathrm{~h}$ and $48 \mathrm{~h}$ after hypoxia. Vehicle-treated animals received sterile saline.

\section{Animal Groups}

The animals were grouped as follows: sham, $n=8$ ( 4 males and 4 females), sham + normothermia + EPO, $n=8$ (4 males and 4 females), sham + hypothermia + EPO, $n=8$ ( 4 males and 4 females), HI + normothermia, $n=26$ (13 males and 13 females), HI + hypothermia, $n=$ 26 (13 males and 13 females), HI + normothermia + EPO, $n=26$ (13 males and 13 females), and HI + hypothermia + EPO, $n=26$ (13 males and 13 females). The numbers of sham-operated animals were limited, because no effects of normothermia and/or EPO were expected in these groups.

\section{Cylinder-Rearing Test}

The CRT was used to assess sensorimotor function (37). At 2 and $5 \mathrm{wk}$ after $\mathrm{HI}$ between 9 and $10 \mathrm{AM}$, animals were individually placed in a Plexiglas transparent cylinder $(2 \mathrm{wk}$ after $\mathrm{HI}: 11 \mathrm{~cm}$ diameter, $30 \mathrm{~cm}$ height; $5 \mathrm{wk}$ after HI: $25 \mathrm{~cm}$ diameter, $30 \mathrm{~cm}$ height) and observed for $3 \mathrm{~min}$. Initial nonimpaired forepaw (left/right/both) preference of weight-bearing contacts during full rear was recorded. The relative nonimpaired forepaw (right, ipsilateral) preference was calculated as $($ right - left $) /($ right + left + both $) \times 100(38)$.

\section{Histology}

Rats were killed at $6 \mathrm{wk}$ of age with pentobarbital $(300 \mathrm{mg} / \mathrm{kg}$ i.p.) and perfused with phosphate-buffered saline followed by $4 \%$ formaldehyde in phosphate-buffered saline. Brains were mounted in paraffin from which coronal sections $(8 \mu \mathrm{m})$ were cut between $\sim 0.28$ and $-3.20 \mathrm{~mm}$ from the bregma. Brain sections were stained with HE and LFB. Both hemispheres (at $\sim 0.28$ and $-3.2 \mathrm{~mm}$ from the bregma) were outlined on full-section images with a Nikon D1 digital camera (Nikon, Tokyo, Japan). Brain areas for HI were outlined manually using Photoshop CS4 software (Adobe Systems, San Jose, CA) and the ratio of ipsilateral to contralateral areas was calculated (16). The relative volumes (as compared with sham-treated animals) of the contralateral cortex (at $\sim 0.28$ and $-3.2 \mathrm{~mm}$ from the bregma) and hippocampus (at approximately $-3.2 \mathrm{~mm}$ from the bregma) were also calculated with the same method. The area of LFB staining in both hemispheres (at $\sim 0.28$ and $-3.2 \mathrm{~mm}$ from the bregma) was quantified using ImageJ 1.42q software (http:// rsb.info.nih.gov/ij/, 1997-2006) and the ratio of ipsilateral to contralateral areas as well as the relative volume (as compared with sham-treated animals) of contralateral areas were also calculated (16). For each animal, six consecutive sections on the same level were cut, and three sections were randomly chosen. A final ratio was obtained after calculation and averaging. We focused on this region of the brain because the effects of $\mathrm{HI}$ are most striking in the hippocampus and overlying cortex in this neonatal HI rat model. This method is widely used to quantify brain damage in such models and has been validated since 1998 (39-44).

\section{Statistics}

All analyses were performed in a blinded setup. Statistical analysis was performed using R (The R Project for Statistical Computing, http:// www.r-project.org) and GraphPad Prism 5 (GraphPad Software, La Jolla, CA) software. Sample size calculation demonstrated that a group size of 13 animals was sufficient to demonstrate differences in CRT of $25 \%$ with an alpha of 0.05 and power of 0.80 while considering multiple comparisons. Data were presented graphically as means \pm SD. Multivariable analysis using general linear modeling was performed with the score on the CRT or histological outcome as dependent variable, and gender, hypothermia, and EPO as independent variables. Interactions were examined.

\section{STATEMENT OF FINANCIAL SUPPORT}

No financial assistance was received to support this study.

\section{REFERENCES}

1. Barnett A, Mercuri E, Rutherford M, et al. Neurological and perceptual-motor outcome at 5-6 years of age in children with neonatal encephalopathy: relationship with neonatal brain MRI. Neuropediatrics 2002;33:242-8.

2. Glass HC, Ferriero DM. Treatment of hypoxic-ischemic encephalopathy in newborns. Curr Treat Options Neurol 2007;9:414-23.

3. Groenendaal F, Brouwer AJ. Clinical aspects of induced hypothermia in full term neonates with perinatal asphyxia. Early Hum Dev 2009;85:73-6.

4. Hosono T, Kamo A, Hakotani S, et al. Effect of hypothermia on motor function of adult rats after neonatal hyperthermic hypoxic-ischemic brain insult. Eur J Appl Physiol 2010;109:35-9.

5. Ohmura A, Nakajima W, Ishida A, et al. Prolonged hypothermia protects neonatal rat brain against hypoxic-ischemia by reducing both apoptosis and necrosis. Brain Dev 2005;27:517-26.

6. Vannucci RC, Towfighi J, Vannucci SJ. Secondary energy failure after cerebral hypoxia-ischemia in the immature rat. J Cereb Blood Flow Metab 2004;24:1090-7.

7. Edwards AD, Brocklehurst P, Gunn AJ, et al. Neurological outcomes at 18 months of age after moderate hypothermia for perinatal hypoxic ischaemic encephalopathy: synthesis and meta-analysis of trial data. BMJ 2010; 340:c363.

8. Gluckman PD, Wyatt JS, Azzopardi D, et al. Selective head cooling with mild systemic hypothermia after neonatal encephalopathy: multicentre randomised trial. Lancet 2005;365:663-70.

9. van Bel F, Groenendaal F. Long-term pharmacologic neuroprotection after birth asphyxia: where do we stand? Neonatology 2008;94:203-10.

10. Sola A, Wen TC, Hamrick SE, Ferriero DM. Potential for protection and repair following injury to the developing brain: a role for erythropoietin? Pediatr Res 2005;57(5 Pt 2):110R-7R.

11. van der Kooij MA, Groenendaal F, Kavelaars A, Heijnen CJ, van Bel F. Neuroprotective properties and mechanisms of erythropoietin in in vitro and in vivo experimental models for hypoxia/ischemia. Brain Res Rev 2008;59:22-33.

12. van der Kooij MA, Groenendaal F, Kavelaars A, Heijnen CJ, van Bel F Combination of deferoxamine and erythropoietin: therapy for hypoxiaischemia-induced brain injury in the neonatal rat? Neurosci Lett 2009;451: 109-13.

13. Fan X, Heijnen CJ, van der Kooij MA, Groenendaal F, van Bel F. Beneficial effect of erythropoietin on sensorimotor function and white matter after hypoxia-ischemia in neonatal mice. Pediatr Res 2011;69:56-61.

14. Zhu C, Kang W, Xu F, et al. Erythropoietin improved neurologic outcomes in newborns with hypoxic-ischemic encephalopathy. Pediatrics 2009;124: e218-26.

15. Bona E, Hagberg H, Løberg EM, Bågenholm R, Thoresen M. Protective effects of moderate hypothermia after neonatal hypoxia-ischemia: shortand long-term outcome. Pediatr Res 1998;43:738-45. 
16. Nijboer CH, Groenendaal F, Kavelaars A, Hagberg HH, van Bel F, Heijnen CJ. Gender-specific neuroprotection by 2-iminobiotin after hypoxia-ischemia in the neonatal rat via a nitric oxide independent pathway. J Cereb Blood Flow Metab 2007;27:282-92.

17. Wen TC, Rogido M, Peng H, Genetta T, Moore J, Sola A. Gender differences in long-term beneficial effects of erythropoietin given after neonatal stroke in postnatal day-7 rats. Neuroscience 2006;139:803-11.

18. Hobbs C, Thoresen M, Tucker A, Aquilina K, Chakkarapani E, Dingley J. Xenon and hypothermia combine additively, offering long-term functional and histopathologic neuroprotection after neonatal hypoxia/ischemia. Stroke 2008;39:1307-13.

19. Fukui O, Kinugasa Y, Fukuda A, et al. Post-ischemic hypothermia reduced IL-18 expression and suppressed microglial activation in the immature brain. Brain Res 2006;1121:35-45.

20. Hasegawa M, Ogihara T, Tamai H, Hiroi M. Hypothermic inhibition of apoptotic pathways for combined neurotoxicity of iron and ascorbic acid in differentiated PC12 cells: reduction of oxidative stress and maintenance of the glutathione redox state. Brain Res 2009;1283:1-13.

21. Guan J, Gunn AJ, Sirimanne ES, et al. The window of opportunity for neuronal rescue with insulin-like growth factor-1 after hypoxia-ischemia in rats is critically modulated by cerebral temperature during recovery. J Cereb Blood Flow Metab 2000;20:513-9.

22. Trescher WH, Ishiwa S, Johnston MV. Brief post-hypoxic-ischemic hypothermia markedly delays neonatal brain injury. Brain Dev 1997;19:326-38.

23. Gonzalez FF, Abel R, Almli CR, Mu D, Wendland M, Ferriero DM. Erythropoietin sustains cognitive function and brain volume after neonatal stroke. Dev Neurosci 2009;31:403-11.

24. Bierer R, Peceny MC, Hartenberger CH, Ohls RK. Erythropoietin concentrations and neurodevelopmental outcome in preterm infants. Pediatrics 2006;118:e635-40.

25. Fauchère JC, Dame C, Vonthein R, et al. An approach to using recombinant erythropoietin for neuroprotection in very preterm infants. Pediatrics 2008;122:375-82.

26. Juul SE, McPherson RJ, Bauer LA, Ledbetter KJ, Gleason CA, Mayock DE. A phase I/II trial of high-dose erythropoietin in extremely low birth weight infants: pharmacokinetics and safety. Pediatrics 2008;122:383-91.

27. Spandou E, Papadopoulou Z, Soubasi V, et al. Erythropoietin prevents long-term sensorimotor deficits and brain injury following neonatal hypoxia-ischemia in rats. Brain Res 2005;1045:22-30.

28. Cariou A, Claessens YE, Pène F, et al. Early high-dose erythropoietin therapy and hypothermia after out-of-hospital cardiac arrest: a matched control study. Resuscitation 2008;76:397-404.
29. Iwai M, Stetler RA, Xing J, et al. Enhanced oligodendrogenesis and recovery of neurological function by erythropoietin after neonatal hypoxic/ischemic brain injury. Stroke 2010;41:1032-7.

30. van den Tweel ER, Kavelaars A, Lombardi MS, et al. Bilateral molecular changes in a neonatal rat model of unilateral hypoxic-ischemic brain damage. Pediatr Res 2006;59:434-9.

31. Du L, Bayir H, Lai Y, et al. Innate gender-based proclivity in response to cytotoxicity and programmed cell death pathway. J Biol Chem 2004;279:38563-70.

32. Zhu C, Xu F, Wang X, et al. Different apoptotic mechanisms are activated in male and female brains after neonatal hypoxia-ischaemia. J Neurochem 2006;96:1016-27.

33. Skoff RP, Bessert D, Barks JD, Silverstein FS. Plasticity of neurons and glia following neonatal hypoxic-ischemic brain injury in rats. Neurochem Res 2007;32:331-42.

34. Johnston MV. Clinical disorders of brain plasticity. Brain Dev 2004;26:73-80.

35. Reitmeir R, Kilic E, Kilic U, et al. Post-acute delivery of erythropoietin induces stroke recovery by promoting perilesional tissue remodelling and contralesional pyramidal tract plasticity. Brain 2011;134(Pt 1):84-99.

36. Rice JE 3rd, Vannucci RC, Brierley JB. The influence of immaturity on hypoxic-ischemic brain damage in the rat. Ann Neurol 1981;9:131-41.

37. Schallert T, Fleming SM, Leasure JL, Tillerson JL, Bland ST. CNS plasticity and assessment of forelimb sensorimotor outcome in unilateral rat models of stroke, cortical ablation, parkinsonism and spinal cord injury. Neuropharmacology 2000;39:777-87.

38. Chang YS, Mu D, Wendland M, et al. Erythropoietin improves functional and histological outcome in neonatal stroke. Pediatr Res 2005;58:106-11.

39. Sheldon RA, Sedik C, Ferriero DM. Strain-related brain injury in neonatal mice subjected to hypoxia-ischemia. Brain Res 1998;810:114-22.

40. Bartley J, Soltau T, Wimborne H, et al. BrdU-positive cells in the neonatal mouse hippocampus following hypoxic-ischemic brain injury. BMC Neurosci 2005;6:15.

41. McAuliffe JJ, Miles L, Vorhees CV. Adult neurological function following neonatal hypoxia-ischemia in a mouse model of the term neonate: water maze performance is dependent on separable cognitive and motor components. Brain Res 2006;1118:208-21.

42. Svedin P, Hagberg H, Mallard C. Expression of MMP-12 after neonatal hypoxic-ischemic brain injury in mice. Dev Neurosci 2009;31:427-36.

43. Yu Z, Li S, Lv SH, et al. Hypoxia-ischemia brain damage disrupts brain cholesterol homeostasis in neonatal rats. Neuropediatrics 2009;40:179-85.

44. Wang X, Han W, Du X, et al. Neuroprotective effect of Bax-inhibiting peptide on neonatal brain injury. Stroke 2010;41:2050-5. 